\title{
Association of High Vitamin D Status with Low Circulating Thyroid-Stimulating Hormone Independent of Thyroid Hormone Levels in Middle-Aged and Elderly Males
}

\author{
Qingqing Zhang, ${ }^{1}$ Zhixiao Wang, ${ }^{1}$ Min Sun, ${ }^{1}$ Mengdie Cao, ${ }^{1}$ Zhenxin Zhu, ${ }^{1}$ \\ Qi Fu, ${ }^{1}$ Yuan Gao, ${ }^{2}$ Jia Mao, ${ }^{1}$ Yanyun Li, ${ }^{1}$ Yun Shi, ${ }^{1}$ Fan Yang, ${ }^{1}$ Shuai Zheng, \\ Wei Tang, ${ }^{2}$ Yu Duan, ${ }^{1}$ Xiaoping Huang, ${ }^{1}$ Wei He, ${ }^{1}$ and Tao Yang ${ }^{1}$ \\ ${ }^{1}$ Department of Endocrinology, The First Affiliated Hospital of Nanjing Medical University, Nanjing, Jiangsu 210029, China \\ ${ }^{2}$ Department of Endocrinology and Metabolism, The Affiliated Jiangyin Hospital of Southeast University Medical College, Jiangyin, \\ Jiangsu 214400, China
}

Correspondence should be addressed to Wei He; hewei1007@sina.cn and Tao Yang; yangt@njmu.edu.cn

Received 5 November 2013; Revised 25 December 2013; Accepted 8 January 2014; Published 16 February 2014

Academic Editor: Zhongjian Xie

Copyright (C) 2014 Qingqing Zhang et al. This is an open access article distributed under the Creative Commons Attribution License, which permits unrestricted use, distribution, and reproduction in any medium, provided the original work is properly cited.

\begin{abstract}
Background. A recent study has reported that high circulating 25-hydroxyvitamin D [25(OH)D] is associated with low circulating thyroid-stimulating hormone (TSH) levels, but only in younger individuals. The goal of the present study was to explore the relationship between vitamin D status and circulating TSH levels with thyroid autoimmunity and thyroid hormone levels taken into consideration in a population-based health survey of middle-aged and elderly individuals. Methods. A total of 1,424 Chinese adults, aged 41-78 years, were enrolled in this cross-sectional study. Serum levels of 25(OH)D, TSH, thyroid hormones, and thyroid autoantibodies were measured. Results. The prevalence of vitamin D insufficiency was $94.29 \%$ in males and $97.22 \%$ in females, and the prevalence of vitamin D deficiency was $55.61 \%$ in males and $69.64 \%$ in females. Vitamin D status was not associated with positive thyroid autoantibodies after controlling for age, gender, body mass index, and smoking status. Higher 25(OH)D levels were associated with lower TSH levels after controlling for age, FT4 and FT3 levels, thyroid volume, the presence of thyroid nodule(s), and smoking status in males. Conclusion. High vitamin D status in middle-aged and elderly males was associated with low circulating TSH levels independent of thyroid hormone levels.
\end{abstract}

\section{Introduction}

Vitamin D is recognized to be an essential element for bone metabolism and skeletal health; however, its deficiency can cause rickets in children as well as an increased propensity for osteoporosis [1]. In addition, it may also affect extraskeletal health. Indeed, vitamin D deficiency has been identified as a risk factor for diabetes mellitus [2, 3], cancers [4], multiple sclerosis [5] and other autoimmune diseases $[6,7]$, atherosclerosis [8], and infectious diseases [9].

Few past studies have reported the impact of vitamin D deficiency on autoimmune thyroid disease and demonstrated inconclusive results $[10,11]$. Besides affecting the thyroid gland through immune-mediated processes, vitamin D has been shown to influence rat thyroid follicular cells by directly inhibiting thyrotropin-stimulated iodide uptake in a dosedependent manner [12]. Recently, a population-based study has reported that high vitamin D status in younger individuals is associated with low circulating thyroid-stimulating hormone (TSH) [13]. However, it remains unknown as to why no relationship between vitamin $\mathrm{D}$ status and serum TSH levels in middle-aged and elderly individuals was found in this study. Therefore, in the present study, we examined the relationship between vitamin $\mathrm{D}$ status and circulating TSH levels in middle-aged and elderly individuals with thyroid autoimmunity, while taking thyroid function into considerationin addition to the relationship between vitamin $\mathrm{D}$ insufficiency and thyroid autoimmunity, the presence of 
thyroid nodule(s) and thyroid volume in a cross-sectional study.

\section{Materials and Methods}

2.1. Study Subjects. From June to December 2011, we recruited a total of 9,982 Chinese living in Gulou, Nanjing. The study was approved by the ethics committee of the First Affiliated Hospital of Nanjing Medical University. All patients provided informed consent and completed a standardized questionnaire. Blood samples were collected and stored at $-80^{\circ} \mathrm{C}$.

The present study used a subsample collected from September to November 2011 to detect serum 25-hydroxyvitamin D [25(OH)D] levels, thyroid function, and thyroid autoantibodies. Subjects with a history of thyroid disease, without complete informations or taking medications that affected their thyroid function, such as oral contraceptives, oestrogen, glucocorticoids and iodine, were excluded.

Further exclusion criteria included overt hypothyroidism (TSH $>5.29 \mu \mathrm{IU} / \mathrm{mL}$; free thyroxine $(\mathrm{FT} 4)<8.5 \mathrm{pmol} / \mathrm{L}$ ) and overt hyperthyroidism (TSH $<0.35 \mu \mathrm{IU} / \mathrm{mL}$; FT4 > $22.5 \mathrm{pmol} / \mathrm{L}$ ). A total of 1,424 participants were included in our study.

2.2. Laboratory Measurements. Serum samples were collected from all 1,424 participants in the morning and all venous blood samples after an overnight fast. The serum samples were used to measure FT4, FT3, TSH, and 25(OH)D levels. Only 1,279 and 1,357 serum samples were used to measure thyroid peroxidase antibody (TPOAb) and thyroglobulin antibody $(\mathrm{TgAb})$, respectively. Serum 25(OH)D levels were assessed using an enzyme immunoassay (IDS, UK). Serum TSH, TPOAb, and TgAb levels were measured using a chemiluminescent immunoassay (AutoBio Co., Ltd., Zhengzhou, China). Euthyroidism was defined as the absence of hypo- or hyperthyroidism. Serum TPOAb of $>40 \mathrm{IU} / \mathrm{mL}$ and/or $\mathrm{TgAb}$ of $>110 \mathrm{IU} / \mathrm{mL}$ were considered autoantibody positivity. High TPOAb and TgAb titres were defined as arbitrary values greater than $200 \mathrm{IU} / \mathrm{mL}$ and $550 \mathrm{IU} / \mathrm{mL}$ (four times greater than the normal values), respectively. We set vitamin $\mathrm{D}$ insufficiency and deficiency at levels below $75 \mathrm{nmol} / \mathrm{L}(30 \mathrm{ng} / \mathrm{mL})$ and $50 \mathrm{nmol} / \mathrm{L}(20 \mathrm{ng} / \mathrm{mL})$, respectively. The presence of thyroid nodule(s) and size of the thyroid gland were determined by thyroid ultrasonography. The thyroid volume was estimated by multiplication of its thickness, width, length, and a corrective factor (0.479) [14].

2.3. Statistical Analysis. Continuous variables are presented as means \pm standard deviation for continuous normally distributed variables and median (interquartile range) for nonnormally distributed variables. Categorical variables are presented as percentage. The Kolmogorov-Smirnov method was used to test for normality. Differences between two groups for continuous and categorical variables were separately assessed using the Student's $t$-test and $\chi^{2}$-test. The distribution of TSH levels deviated significantly from a Gaussian distribution $(P<0.000)$, while the logarithms of TSH levels were found to follow an approximate Gaussian distribution $(P=0.065)$. Linear regression analysis was used to examine the relationship between vitamin D status and $\log$ TSH values. Furthermore, bivariate correlation analysis was performed to study the correlation between vitamin D status and $\log \mathrm{TSH}$ values. All calculations were performed using SPSS 13.0 for Windows (Chicago, IL, USA). A probability $(P)$ value of $<0.05$ was considered statistically significant for all tests.

\section{Results}

3.1. Clinical and Laboratory Characteristics. The clinical and laboratory characteristics of the included 1,424 subjects (863 females and 561 males; age, 41-78 years) are shown in Table 1. On an average, females had higher serum TSH levels and $\mathrm{TgAb}$ titres, but lower serum 25(OH)D levels and proportion of smokers than males (3.03 (range, 1.89-4.60) versus 2.06 (1.36-3.30) IU/mL, $P<0.01 ; 13.19$ (7.90-35.66) versus 10.08 (6.87-19.22) IU/mL, $P<0.01 ; 42.90$ (35.15-53.11) versus 47.50 (38.00-59.00) IU/mL, $P<0.01$ and $1.85 \%$ versus $44.92 \%$, $P<0.01$, resp.). There were no significant differences in other laboratory characteristics between males and females. The overall median (interquartile range) serum $25(\mathrm{OH}) \mathrm{D}$ level was 44.68 (range, 36.2-55.3) nmol/L. The prevalence of vitamin D insufficiency was $94.29 \%$ in males and $97.22 \%$ in females, whereas the prevalence of vitamin D deficiency was $55.61 \%$ in males and $69.64 \%$ in females. In the present study, $138(10.17 \%)$ subjects were positive for serum $\mathrm{TgAb}$, of which $42(30.43 \%)$ had high TgAb titres. A total of $152(11.88 \%)$ subjects were positive for serum TPOAb, of which 55 (36.18\%) had high TPOAb titres. The prevalence of thyroid nodule(s) was $36.03 \%$.

3.2. Comparison of Clinical and Laboratory Characteristics between Vitamin D Insufficiency and Sufficiency Groups. Based on a cutoff value of $75 \mathrm{nmol} / \mathrm{L}(30 \mathrm{ng} / \mathrm{mL})$, the subjects were grouped into vitamin D insufficiency and vitamin D sufficiency. Differences between the clinical and laboratory characteristics between the two groups are shown in Table 2. There were significantly fewer males in the vitamin $D$ insufficiency group (38.67\% versus $57.14 \%, P=0.006)$ than in the vitamin D sufficiency group. The serum TSH level and TPOAb and $\operatorname{TgAb}$ titres in the vitamin D insufficiency group were significantly higher than those in the vitamin $\mathrm{D}$ sufficiency group. However, there were no significant differences in serum FT3 and FT4 levels, the presence of thyroid nodule(s), thyroid volume, and smoking status between the two groups. Multivariate logistic regression analysis revealed that vitamin D status was not associated with thyroid autoantibody positivity after controlling for age, gender, body mass index (BMI), and smoking status (Table 3). Based on a cutoff value of $50 \mathrm{nmol} / \mathrm{L}(20 \mathrm{ng} / \mathrm{mL})$, the subjects were grouped into vitamin D deficiency and nonvitamin D deficiency. High TgAb titres, High TPOAb titres, and high $\mathrm{TgAb}$ and/or TPOAb titres were significantly more common in the vitamin $\mathrm{D}$ deficiency group compared with those in the nonvitamin D deficiency group $(4.04 \%$ versus $1.43 \%, P=$ $0.008 ; 5.48 \%$ versus $2.18 \%, P=0.005 ; 7.70 \%$ versus $3.60 \%, P=$ 0.004 , resp.). And vitamin D deficiency was independently 
TABLE 1: Clinical and laboratory characteristics of the participants.

\begin{tabular}{lccc}
\hline Characteristics & Male $(n=561)$ & Female $(n=863)$ & Total $(n=1424)$ \\
\hline Age $($ years $)$ & $59.22 \pm 9.03$ & $59.04 \pm 8.20$ & $59.11 \pm 8.53$ \\
BMI $\left(\mathrm{kg} / \mathrm{m}^{2}\right)$ & $24.93 \pm 3.38$ & $24.66 \pm 3.36$ & $24.76 \pm 3.37$ \\
Serum $25(\mathrm{OH}) \mathrm{D}(\mathrm{nmol} / \mathrm{L})$ & $47.50(38.00-59.00)$ & $42.90(35.15-53.11)^{*}$ & $44.68(36.20-55.30)$ \\
Serum FT3 $(\mathrm{pmol} / \mathrm{L})$ & $4.34(3.99-4.69)$ & $4.31(3.98-4.72)$ & $4.32(3.98-4.69)$ \\
Serum FT4 $(\mathrm{pmol} / \mathrm{L})$ & $15.87(13.10-18.03)$ & $15.81(13.22-17.95)$ & $15.83(13.18-17.97)$ \\
Serum TSH $(\mu \mathrm{IU} / \mathrm{mL})$ & $2.06(1.36-3.30)$ & $3.03(1.89-4.60)^{*}$ & $2.65(1.62-4.10)$ \\
Serum TPOAb $(\mathrm{IU} / \mathrm{mL})$ & $3.09(1.07-11.08)$ & $4.18(1.24-16.28)$ & $3.66(1.15-14.34)$ \\
Serum TgAb $(\mathrm{IU} / \mathrm{mL})$ & $10.08(6.87-19.22)$ & $13.19(7.90-35.66)^{*}$ & $12.06(7.31-27.00)$ \\
Thyroid volume $(\mathrm{mL})$ & $8.44(6.82-10.06)$ & $7.24(5.83-8.82)$ & $7.74(6.16-9.40)$ \\
Presence of thyroid nodule $(\mathrm{s})(n(\%))$ & $174(31.02 \%)$ & $339(39.28 \%)^{*}$ & $513(36.03 \%)$ \\
Current smokers $(n(\%))$ & $252(44.92 \%)$ & $16(1.85 \%)^{*}$ & $268(18.82 \%)$ \\
\hline
\end{tabular}

${ }^{*}$ Significantly different from males, $P<0.01$.

TABLE 2: Comparison of clinical and laboratory characteristics based on vitamin D insufficiency.

\begin{tabular}{lccc}
\hline & Vitamin D insufficiency $(1368)$ & Nonvitamin D insufficiency (56) & $P$ value \\
\hline Age $($ years $)$ & $59.07 \pm 8.55$ & $60.07 \pm 8.11$ & 0.371 \\
Male sex $(n(\%))$ & $529(38.67 \%)$ & $32(57.14 \%)$ & 0.006 \\
BMI $\left(\mathrm{kg} / \mathrm{m}^{2}\right)$ & $24.79 \pm 3.40$ & $24.21 \pm 2.50$ & 0.106 \\
Serum FT3 $(\mathrm{pmol} / \mathrm{L})$ & $4.39(3.97-4.69)$ & $4.48(4.18-4.75)$ & 0.094 \\
Serum FT4 $(\mathrm{pmol} / \mathrm{L})$ & $15.51(13.14-17.93)$ & $16.29(14.01-18.79)$ & 0.092 \\
Serum TSH $(\mu \mathrm{IU} / \mathrm{mL})$ & $3.42(1.62-4.18)$ & $2.47(1.54-3.24)$ & 0.000 \\
Serum TgAb $(\mathrm{IU} / \mathrm{mL})$ & $84.26(7.26-27.77)$ & $21.12(8.38-22.40)$ & 0.000 \\
Serum TPOAb $(\mathrm{IU} / \mathrm{mL})$ & $40.45(1.17-14.33)$ & $16.77(0.99-14.99)$ & 0.000 \\
Thyroid volume $(\mathrm{mL})$ & $8.43(6.12-9.35)$ & $8.71(6.53-10.62)$ & 0.476 \\
Presence of thyroid nodule $(\mathrm{s})(n(\%))$ & $495(36.18 \%)$ & $18(32.14 \%)$ & 0.537 \\
Current smokers $(n(\%))$ & $814(59.50 \%)$ & $37(66.07 \%)$ & 0.326 \\
\hline
\end{tabular}

associated with high thyroid autoantibody titres (see Supplementary Table 1 in the Supplementary Material available online at http://dx.doi.org/10.1155/2014/631819).

3.3. Relationship between Vitamin D Status and Serum TSH Levels according to Gender. With regard to the relationship between vitamin D status and serum TSH level, it was found that higher $25(\mathrm{OH}) \mathrm{D}$ levels were associated with lower TSH levels independent of age, FT3 and FT4 levels, TPOAb and $\mathrm{TgAb}$ titres, thyroid volume, the presence of thyroid nodule(s), and smoking status in males (Beta $=-0.166$, $P=0.004$; Table 4). In addition, the association was found between thyroid volume and serum TSH levels in males (Beta $=-0.152, P=0.009$ ) as well as the presence of thyroid nodule(s) and serum TSH levels in females (Beta $=-0.116$, $P=0.023)$. However, there were no significant relationship between serum TSH, FT3 and FT4 levels, TgAb and TPOAb titres, and smoking status between genders.

3.4. Relationship between Vitamin D Status and Serum TSH Levels on the Basis of Negative Serum Thyroid Autoantibody Titres in Males. To determine the probable interaction between vitamin $\mathrm{D}$ status and thyroid autoantibody positivity on serum TSH levels, further analyses were performed based on positive serum TPOAb, TgAb and TPOAb, and/or TgAb titres. The concentration of $25(\mathrm{OH}) \mathrm{D}$ was negatively correlated with serum TSH levels only in subjects with negative serum TPOAb, TgAb and TPOAb and/or TgAb titres $(P=$ 0.002, 0.005 and 0.002, resp.; Supplementary Table 2). Linear regression analysis showed that higher $25(\mathrm{OH}) \mathrm{D}$ levels were associated with lower TSH levels independent of age, serum thyroid hormone levels, thyroid volume, the presence of thyroid nodule(s), and smoking status in males with negative serum TPOAb, TgAb, and TPOAb and/or TgAb titres $(P=$ $0.004,0.004$, and 0.002 , resp.; Table 5), but not in females or subjects positive for serum thyroid autoantibodies (data not shown).

\section{Discussion}

In the present study, we explored the probable interaction between vitamin $\mathrm{D}$ status and thyroid autoantibodies on serum TSH levels in middle-aged and elderly Chinese population with euthyroidism. This report is the first, to the best of our knowledge, to show that a higher circulating $25(\mathrm{OH}) \mathrm{D}$ level was associated with lower TSH levels only in males with negative serum thyroid autoantibody titres independent of thyroid hormone levels. 
TABLE 3: Determinants of positive serum thyroid antibodies.

\begin{tabular}{|c|c|c|c|c|c|c|}
\hline & \multicolumn{2}{|l|}{ Serum TgAb } & \multicolumn{2}{|l|}{ Serum TPOAb } & \multicolumn{2}{|c|}{ Serum $\mathrm{TgAb}$ and/or TPOAb } \\
\hline & Adjusted OR (95\% CI) & $P$ value & Adjusted OR (95\% CI) & $P$ value & Adjusted OR (95\% CI) & $P$ value \\
\hline Age (years) & $0.997(0.974-1.022)$ & 0.838 & $1.007(0.983-1.032)$ & 0.558 & $1.009(0.987-1.030)$ & 0.431 \\
\hline Male sex & $2.738(1.526-4.910)$ & 0.001 & $2.200(1.267-3.821)$ & 0.005 & $2.524(1.550-4.110)$ & 0.000 \\
\hline BMI $\left(\mathrm{kg} / \mathrm{m}^{2}\right)$ & $1.035(0.974-1.099)$ & 0.266 & $1.040(0.979-1.106)$ & 0.204 & $1.039(0.984-1.097)$ & 0.166 \\
\hline Smoking status & $0.786(0.368-1.676)$ & 0.532 & $1.308(0.685-2.499)$ & 0.415 & $1.052(0.578-1.916)$ & 0.868 \\
\hline Serum $25(\mathrm{OH}) \mathrm{D}(\mathrm{nmol} / \mathrm{L})$ & $0.992(0.979-1.004)$ & 0.188 & $0.999(0.987-1.010)$ & 0.818 & $0.997(0.986-1.009)$ & 0.660 \\
\hline
\end{tabular}

TABLE 4: Standardized regression coefficients of variables in relation to serum TSH according to gender.

\begin{tabular}{|c|c|c|c|c|}
\hline & \multicolumn{2}{|c|}{ Male (561) } & \multicolumn{2}{|c|}{ Female (863) } \\
\hline & Beta & $P$ value & Beta & $P$ value \\
\hline Age (years) & -0.012 & 0.836 & -0.023 & 0.647 \\
\hline Serum FT3 (pmol/L) & 0.069 & 0.244 & -0.043 & 0.399 \\
\hline Serum FT4 (pmol/L) & -0.040 & 0.501 & -0.066 & 0.201 \\
\hline Presence of $\operatorname{TgAb}(\%)$ & 0.029 & 0.636 & 0.098 & 0.060 \\
\hline Presence of TPOAb (\%) & -0.002 & 0.971 & 0.056 & 0.284 \\
\hline Thyroid volume (mL) & -0.152 & 0.009 & -0.056 & 0.262 \\
\hline $\begin{array}{l}\text { Presence of thyroid } \\
\text { nodule(s) (\%) }\end{array}$ & -0.025 & 0.669 & -0.116 & 0.023 \\
\hline Current smokers $(n(\%))$ & -0.054 & 0.361 & -0.001 & 0.985 \\
\hline Serum 25(OH)D (nmol/L) & -0.166 & 0.004 & -0.001 & 0.983 \\
\hline
\end{tabular}

TABLE 5: Standardized regression coefficients of variables in relation to serum TSH in males with negative serum antibodies.

\begin{tabular}{lcccccc}
\hline & \multicolumn{2}{c}{ Serum TgAb } & \multicolumn{2}{c}{ Serum TPOAb } & \multicolumn{2}{c}{ Serum TgAb and/or TPOAb } \\
& Beta & $P$ value & Beta & $P$ value & Beta & $P$ value \\
\hline Age (years) & 0.024 & 0.686 & 0.019 & 0.749 & 0.006 & 0.921 \\
Serum FT3 (pmol/L) & 0.030 & 0.593 & 0.092 & 0.122 & 0.074 & 0.228 \\
Serum FT4 (pmol/L) & 0.020 & 0.726 & -0.037 & 0.535 & -0.028 & 0.648 \\
Thyroid volume (mL) & -0.165 & 0.003 & -0.170 & 0.004 & -0.154 & 0.011 \\
Presence of thyroid nodule(s) (\%) & -0.035 & 0.532 & -0.020 & 0.736 & -0.024 & 0.696 \\
Current smokers $(n(\%))$ & -0.064 & 0.264 & -0.060 & 0.313 & -0.067 & 0.277 \\
Serum 25(OH)D (nmol/L) & -0.157 & 0.004 & -0.166 & 0.004 & -0.184 & 0.002 \\
\hline
\end{tabular}

The result that high vitamin D status was associated with low circulating TSH levels remained unknown. We hypothesized that vitamin D may influence the thyrotrophs by acting on vitamin $\mathrm{D}$ receptors, which are widely distributed through distinct portions of the brain system [15]. A previous study has showed that vitamin $\mathrm{D}$ modulated pituitary thyrotropin TSH secretion by binding to specific binding sites [16]. Smith et al. [17] also found that exogenous vitamin $\mathrm{D}$ administration significantly suppressed pituitary thyrotropin TSH secretion in the basal state. This study also found that serum TSH levels of middle-aged and elderly women were higher than those of same-age men, and this result was consistent with those of previous reports [18, 19]. This result may indicate that TSH secretion is regulated by sex hormones, genetic susceptibility, or environmental factors, which may also mediate the relationship between vitamin $\mathrm{D}$ status and serum TSH levels. Another previous study [20] has showed that circulating oestrogen could induce acute serum TSH suppression in males by acting on the pituitary gland, and vitamin D was shown to be an important factor in oestrogen biosynthesis of both female and male gonads [21]. However, oestrogen administration has been reported to both increase [22] and decrease [23] or have no effect on thyroid activity in females [24]. Therefore, it may be safely inferred that oestrogens have a complex relationship with serum TSH secretion in females, which may explain the cause of this relationship only in men. We did not find the relationship between vitamin D status and serum TSH levels in subjects with positive thyroid antibody titres, which may be due to the limited number of participants, especially when divided into two groups according to gender. Further clinical investigation with a larger sample is required to elucidate the effects of 
vitamin $\mathrm{D}$ on the thyrotrophs to provide further insight into this relationship.

Chailurkit et al. [13] reported that an increase in serum $25(\mathrm{OH}) \mathrm{D}$ concentration was independently associated with lower TSH, but only in younger (age, 15-44 years) individuals. However, we found that higher $25(\mathrm{OH}) \mathrm{D}$ levels were independently associated with lower TSH in males with euthyroidism aged over 40 years. The results of the present study and those of Chailurkit et al's study differed for some reasons. First, subjects with overt hyperthyroidism and hypothyroidism by measuring serum FT3, FT4 and TSH levels were excluded in our study, and the results of our study reflect the general population setting with euthyroidism. Second, Chailurkit et al. did not separately analyse the relationship between $25(\mathrm{OH}) \mathrm{D}$ and serum TSH levels in males and females. Third, thyroid volume, the presence of thyroid nodule(s), and smoking status were considered confounding factors in our statistical analyses. Lastly, ethnicity was previously reported to be related with TSH levels [18], which plausibly explained the difference between the present and Chailurkit's studies.

It is generally agreed that serum $25(\mathrm{OH}) \mathrm{D}$ levels of $<75 \mathrm{nmol} / \mathrm{L}(30 \mathrm{ng} / \mathrm{mL}$ ) should be considered as representative of vitamin D insufficiency, whereas serum 25(OH)D levels of $<50 \mathrm{nmol} / \mathrm{L}(20 \mathrm{ng} / \mathrm{mL})$ as an indicative of vitamin $\mathrm{D}$ deficiency [25]. Using these definitions, our data showed that the prevalence of vitamin D insufficiency was $94.3 \%$ in males and $97.3 \%$ in females, and the prevalence of vitamin $\mathrm{D}$ deficiency was $55.6 \%$ in males and $69.6 \%$ in females. However, there were no conclusive results on the impact of vitamin D deficiency on autoimmune thyroid disease $[18,26]$. In addition, our study did not find a link between vitamin D status and thyroid autoantibody positivity after controlling for age, gender, BMI, and smoking status. However, vitamin $\mathrm{D}$ deficiency was independently associated with high-titre thyroid autoantibody positivity. We thought that this hightitre thyroid autoantibody positivity should be paid more attention.

There were a number of limitations to the present study. First, TSH receptor-stimulating antibodies were not measured. Second, our study focused on middle-aged and elderly individuals; therefore, the relationship between vitamin D status and serum TSH levels in younger individuals with negative serum thyroid autoantibodies remained unknown. Finally, because of the cross-sectional nature of the present study, the causative effect of vitamin D on serum TSH could not be readily determined.

\section{Conclusion}

There was a high prevalence of vitamin D insufficiency among healthy adults. This population-based study was the first, to the best of our knowledge, to report an association between vitamin $\mathrm{D}$ and serum TSH levels independent of thyroid hormone levels in middle-aged and elderly males with negative thyroid autoimmunity. Here, we demonstrated a link between vitamin D insufficiency and serum thyroid autoantibody levels; however, vitamin D status was not associated with positive thyroid autoantibody titres after controlling for age, gender, and smoking status. Therefore, further longitudinal studies are required to clarify the relationship between vitamin D and serum TSH levels, particularly in subjects with negativeserum thyroid autoantibody titres.

\section{Conflict of Interests}

The authors declare that there is no conflict of interests regarding the publication of this paper.

\section{Authors' Contribution}

Qingqing Zhang, Zhixiao Wang, and Min Sun contributed equally to this study.

\section{Acknowledgments}

The authors thank all the participants in this study. This work was supported by the grants from the Chinese Society of Endocrinology and the Project Funded by Jiangsu Provincial Special Program of Medical Science (BL2012026).

\section{References}

[1] M. F. Holick, "Medical progress: vitamin D deficiency," The New England Journal of Medicine, vol. 357, no. 3, pp. 266-281, 2007.

[2] A. G. Pittas, J. Lau, F. B. Hu, and B. Dawson-Hughes, "Review: the role of vitamin $\mathrm{D}$ and calcium in type 2 diabetes. A systematic review and meta-analysis," Journal of Clinical Endocrinology and Metabolism, vol. 92, no. 6, pp. 2017-2029, 2007.

[3] J. Mitri, M. D. Muraru, and A. G. Pittas, "Vitamin D and type 2 diabetes: a systematic review," European Journal of Clinical Nutrition, vol. 65, no. 9, pp. 1005-1015, 2011.

[4] C. F. Garland, E. D. Gorham, S. B. Mohr, and F. C. Garland, "Vitamin D for cancer prevention: global perspective," Annals of Epidemiology, vol. 19, no. 7, pp. 468-483, 2009.

[5] K. L. Munger, S. M. Zhang, E. O’Reilly et al., "Vitamin D intake and incidence of multiple sclerosis," Neurology, vol. 62, no. 1, pp. 60-65, 2004.

[6] M. Cutolo and K. Otsa, "Vitamin D, immunity and lupus," Lupus, vol. 17, no. 1, pp. 6-10, 2008.

[7] M. F. Holick, "Vitamin D: extraskeletal health," Endocrinology and Metabolism Clinics of North America, vol. 39, no. 2, pp. 381400, 2010.

[8] T. J. Wang, M. J. Pencina, S. L. Booth et al., "Vitamin D deficiency and risk of cardiovascular disease," Circulation, vol. 117, no. 4, pp. 503-511, 2008.

[9] J. J. Cannell, R. Vieth, J. C. Umhau et al., "Epidemic influenza and vitamin D," Epidemiology and Infection, vol. 134, no. 6, pp. 1129-1140, 2006.

[10] G. Tamer, S. Arik, I. Tamer, and D. Coksert, "Relative vitamin D insufficiency in Hashimoto's thyroiditis," Thyroid, vol. 21, no. 8, pp. 891-896, 2011.

[11] H. Orbach and Y. Shoenfeld, "Vaccination infection and autoimmunity: myth and reality VIAMR 2005-10-26-28, BeauRivage Palace Hotel, Lausanne, Switzerland," Autoimmunity Reviews, vol. 6, no. 5, pp. 261-266, 2007.

[12] J. P. Berg, K. M. Liane, S. B. Bjorhovde, T. Bjoro, P. A. Torjesen, and E. Haug, "Vitamin D receptor binding and biological effects 
of cholecalciferol analogues in rat thyroid cells," Journal of Steroid Biochemistry and Molecular Biology, vol. 50, no. 3-4, pp. 145-150, 1994.

[13] L. O. Chailurkit, W. Aekplakorn, and B. Ongphiphadhanakul, "High vitamin D status in younger individuals is associated with low circulating thyrotropin," Thyroid, vol. 23, no. 1, pp. 25-30, 2013.

[14] W. Teng, Z. Shan, X. Teng et al., "Effect of iodine intake on thyroid diseases in China," The New England Journal of Medicine, vol. 354, no. 26, pp. 2783-2793, 2006.

[15] K. Prüfer, T. D. Veenstra, G. F. Jirikowski, and R. Kumar, "Distribution of 1,25-dihydroxyvitamin D3 receptor immunoreactivity in the rat brain and spinal cord," Journal of Chemical Neuroanatomy, vol. 16, no. 2, pp. 135-145, 1999.

[16] M. Sar, W. E. Stumpf, and H. F. DeLuca, "Thyrotropes in the pituitary are target cells for 1,25 dihydroxy vitamin D3," Cell and Tissue Research, vol. 209, no. 1, pp. 161-166, 1980.

[17] M. A. Smith, C. McHenry, R. Oslapas, C. Hofmann, P. Hessel, and E. Paloyan, "Altered TSH levels associated with increased serum 1,25-dihydroxyvitamin D3: a possible link between thyroid and parathyroid disease," Surgery, vol. 106, no. 6, pp. 987-991, 1989.

[18] J. G. Hollowell, N. W. Staehling, W. Dana Flanders et al., "Serum TSH, T4, and thyroid antibodies in the United States population (1988 to 1994): national Health and Nutrition Examination Survey (NHANES III)," Journal of Clinical Endocrinology and Metabolism, vol. 87, no. 2, pp. 489-499, 2002.

[19] C. Li, H. Guan, X. Teng et al., "An epidemiological study of the serum thyrotropin reference range and factors that influence serum thyrotropin levels in iodine sufficient areas of China," Endocrine Journal, vol. 58, no. 11, pp. 995-1002, 2011.

[20] H. A. Gross, M. D. Appleman Jr., and J. T. Nicoloff, "Effect of biologically active steroids on thyroid function in man," Journal of Clinical Endocrinology and Metabolism, vol. 33, no. 2, pp. 242248, 1971.

[21] K. Kinuta, H. Tanaka, T. Moriwake, K. Aya, S. Kato, and Y. Seino, "Vitamin D is an important factor in estrogen biosynthesis of both female and male gonads," Endocrinology, vol. 141, no. 4, pp. 1317-1324, 2000.

[22] T. Yamada, Y. Takemura, I. Kobayashi, and K. Shichijo, "Reevaluation of the effect of estrogen on thyroid activity in the rat and its mechanism," Endocrinology, vol. 79, no. 5, pp. 849-857, 1966.

[23] E. M. Bogdanove and B. A. Eskin, "The influence of estrogen upon goiter induction in adult and immature rats," Endocrinology, vol. 59, no. 6, pp. 688-694, 1956.

[24] D. Christianson, E. Roti, A. G. Vagenakis, and L. E. Braverman, "The sex-related difference in serum thyrotropin concentration is androgen mediated," Endocrinology, vol. 108, no. 2, pp. 529535, 1981.

[25] M.-C. Chapuy, P. Preziosi, M. Maamer et al., "Prevalence of vitamin D insufficiency in an adult normal population," Osteoporosis International, vol. 7, no. 5, pp. 439-443, 1997.

[26] R. Goswami, R. K. Marwaha, N. Gupta et al., "Prevalence of vitamin D deficiency and its relationship with thyroid autoimmunity in Asian Indians: a community-based survey," British Journal of Nutrition, vol. 102, no. 3, pp. 382-386, 2009. 


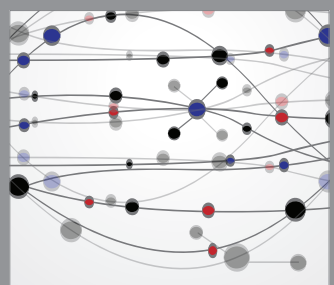

The Scientific World Journal
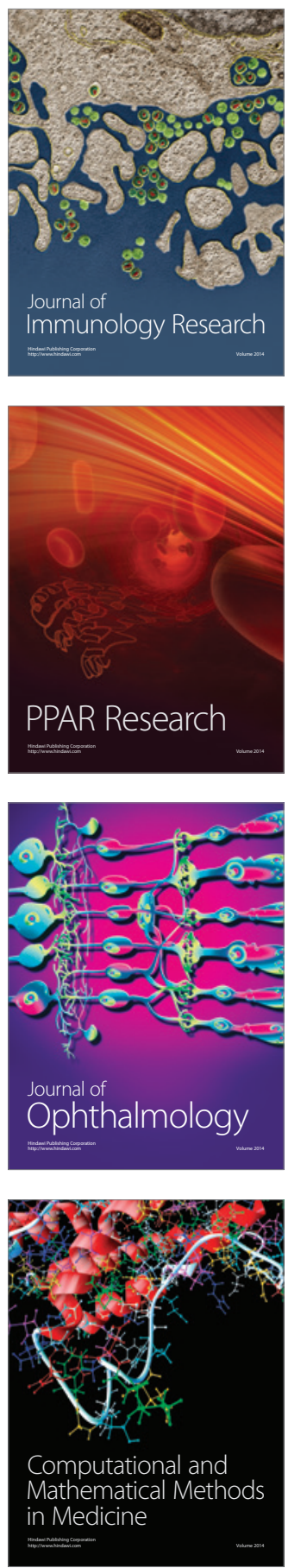

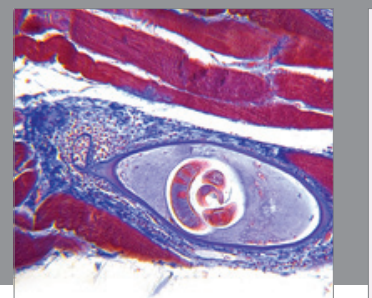

Gastroenterology

Research and Practice
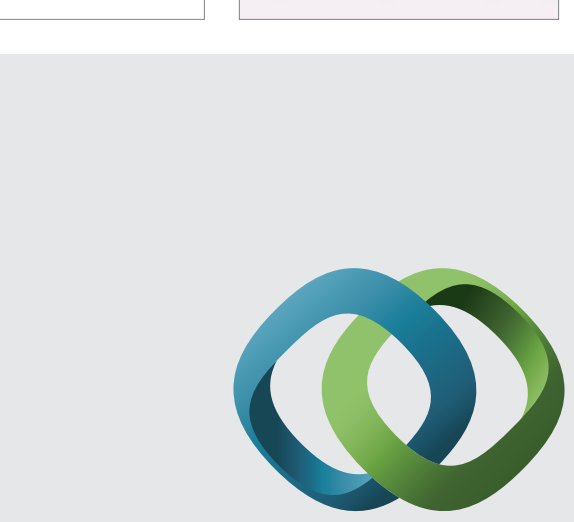

\section{Hindawi}

Submit your manuscripts at

http://www.hindawi.com
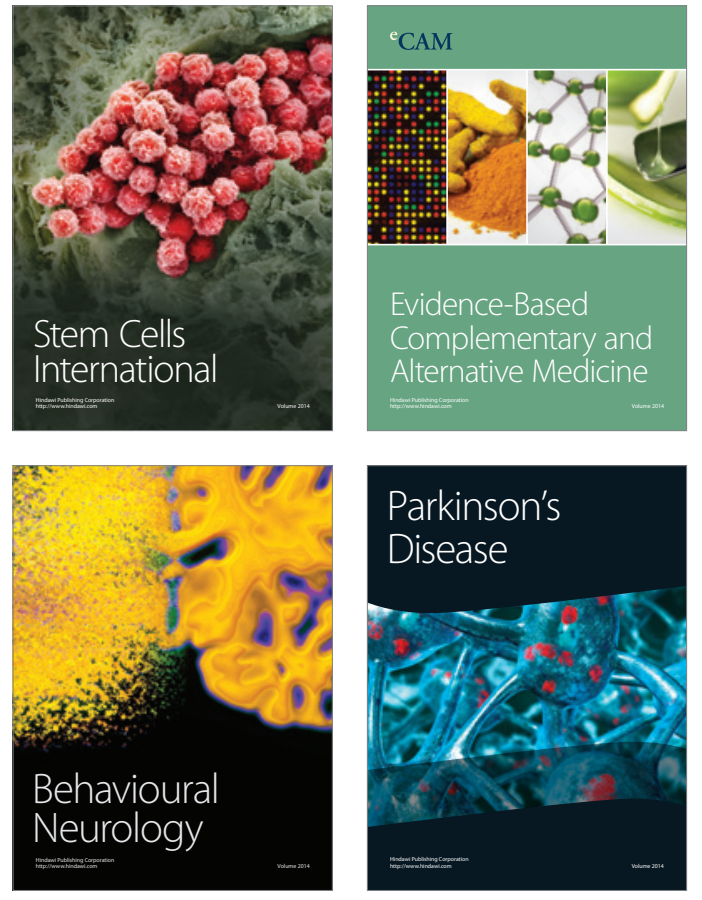
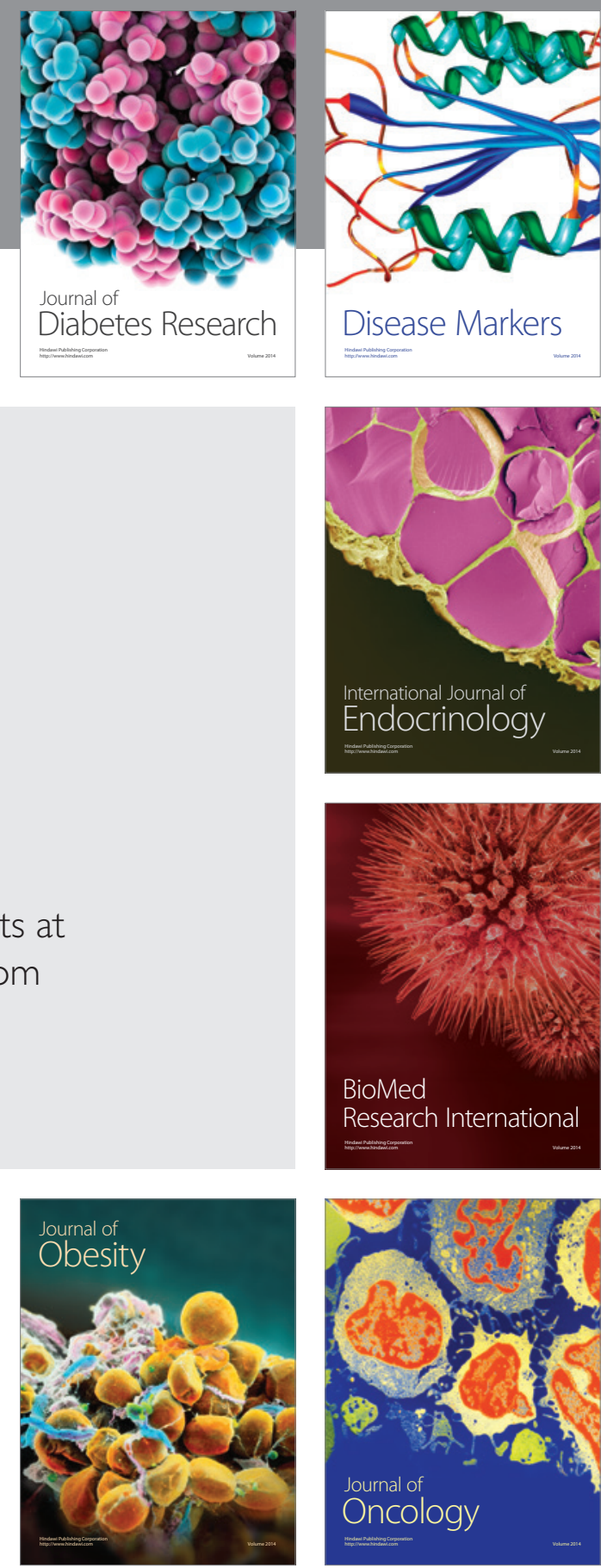

Disease Markers
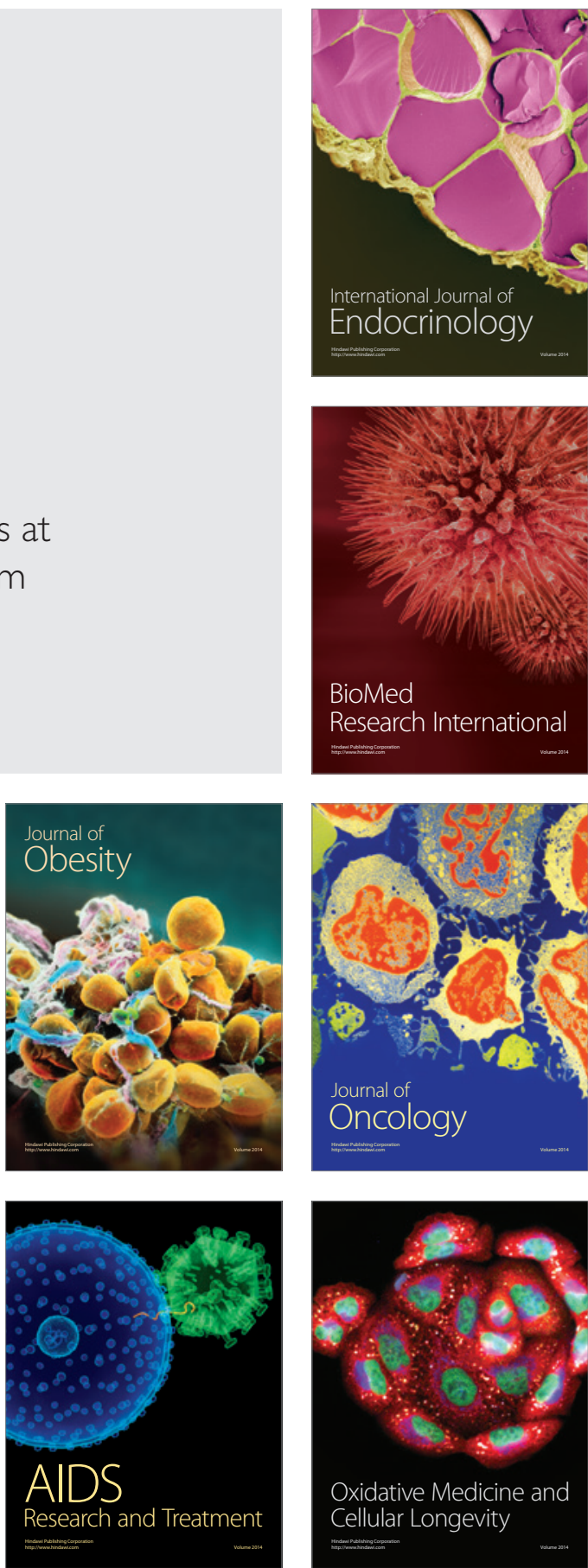